\title{
Trastorno obsesivo compulsivo con tics motores y verbales, trastorno de acumulación y síndrome del acento extranjero sin afasia: comunicación de un caso y revisión bibliográfica.
}

Obsessive compulsive disorder with motor and vocal tics, hoarding behaviour and foreign accent syndrome without aphasia: a case report and literature review.

\author{
Fernando Lázaro-Perlado ${ }^{\text {a, b }}$, Oihane Mentxaka-Solozabal a, Elena Marín-Diaz-Guardamino a , \\ Manuel Conde-Rivas a ${ }^{\text {, Paula Erazo-Presser }}{ }^{\text {b }}$, Zuberoa Maite Miranda-Artieda ${ }^{\text {b. }}$. \\ ${ }^{a}$ Osakidetza - Servicio Vasco de Salud. Red de Salud Mental de Bizkaia. Hospital de Bermeo, Servicio de \\ Rehabilitación, Bermeo, Bizkaia, España ${ }^{b}$ Osakidetza-Servicio Vasco de Salud. Hospital Górliz, Unidad de \\ Rehabilitación de Daño Cerebral, Gorliz, Bizkaia, España.
}

Correspondencia: Fernando Lázaro-Perlado (fernando.lazaroperlado@osakidetza.net)

Recibido: 17/12/2012; aceptado con modificaciones: 08/04/2013

\begin{abstract}
RESUMEN: Informamos sobre un caso de trastorno obsesivo compulsivo (TOC), con tics motores y verbales, conducta acumuladora y síndrome del acento extranjero (dialecto regional) sin afasia, en un nativo español diestro que presentó una hemorragia en ganglios basales izquierdos. Su TOC y conducta acumuladora, que precedieron a la hemorragia cerebral, se diagnosticaron a raíz de un ingreso hospitalario, ya que previamente no había estado en contacto con ningún servicio psiquiátrico. Una lesión limitada al núcleo estriado izquierdo le provocó una variante regional del síndrome del acento extranjero (SAE). El paciente respondió adecuadamente al tratamiento. En nuestro conocimiento, éste es el primer caso de SAE en un hispanoparlante y también en un individuo que presentaba previamente TOC, tics y conducta acumuladora. Revisamos la literatura científica de estos trastornos. PALABRAS CLAVE: trastorno obsesivo compulsivo con tics; trastorno por acumulación; síndrome del acento extranjero.
\end{abstract}

\begin{abstract}
We report a case of obsessive-compulsive disorder (OCD), with motor and vocal tics, hoarding behaviour and foreign accent syndrome (FAS) (regional dialect) without aphasia in a right handed native Spaniard who presented with a left basal ganglia haemorrhage. His OCD and hoarding behaviour, which predated the brain haemorrhage, were diagnosed as a result of his admission to hospital as he had never been in contact with psychiatric services. Limited damage to the left striatum caused a regional variant of foreign accent syndrome. The patient responded well to treatment. To our knowledge this is the first case of a regional dialect of FAS in a Spanish speaker and also in a subject that presented OCD, tics and hoarding behaviour. We review the literature of these disorders.

KEY WORDS: Obsessive compulsive disorder with tics; hoarding; foreign accent syndrome.
\end{abstract}

\section{Agradecimientos:}

Nos gustaría dar las gracias a la Sra. Marijo García, del Departamento de Informática del Hospital de Bermeo por su amable ayuda en la preparación de este manuscrito. 
ORIGINALES Y REVISIONES

\section{Presentación del caso clínico}

Presentamos el caso de un varón diestro de 46 años que acudió a su hospital comarcal con una hemiplejia derecha. En el TAC cerebral que se le realizó se objetivó un hematoma en hemisferio cerebral izquierdo, que afectaba al núcleo estriado y a la cápsula interna. La administración de contraste intravenoso descartó la existencia de un sangrado activo, así como alteraciones estructurales del Polígono de Willis u otras malformaciones arterio-venosas que pudieran justificar la hemorragia. Sin embargo, se detectó una hipoplasia de la arteria vertebral izquierda (Fig. 1). Fue diagnosticado de hipertensión arterial, para la que recibió el oportuno tratamiento farmacológico, y una vez estabilizado se le derivó a nuestro servicio de rehabilitación.

Figura 1

Tomografía axial computarizada (TAC) del cerebro, sin contraste, que muestra una hemorragia capsulo-estriada izquierda (que mide $25 \times 38 \times 47 \mathrm{~mm}$ ) con un borrado de los surcos del lado izquierdo y un mínimo desplazamiento de la línea media.

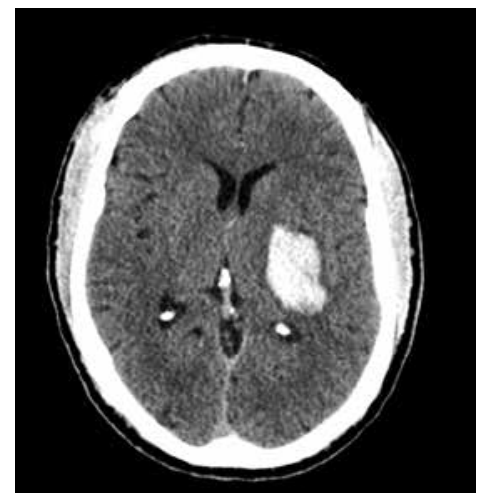

El paciente fue remitido para valoración neuropsiquiátrica ya que su discurso resultaba llamativamente sobre-inclusivo y se mostraba excesivamente preocupado con su programa de rehabilitación y su evolución.

Antes de la primera entrevista con el paciente tuvimos la oportunidad de hablar con su hermano. Éste nos comunicó que tras recibir noticias del hospital acudió al piso del paciente para recoger algunas pertenencias personales del mismo, encontrándose con una vivienda prácticamente llena de libros, DVDs, utensilios de bricolaje y reproductores de DVD. Quedaba un estrecho pasillo que conectaba el dormitorio con la cocina y baño. En un reconocimiento más detallado, observó que su hermano poseía 3 o 4 copias de los mismos libros. También remarcó que tenía muchas prendas de ropa, pero del mismo tipo (por ejemplo, mismo tipo y color de 
ORIGINALES Y REVISIONES

pantalones o camisas...) y unos 20 pares de zapatos idénticos. También vio muchos juguetes que había conservado de su infancia y que se encontraban en perfecto estado. Aunque abarrotado, el piso estaba extremadamente limpio.

A través de lo referido por su hermano, supimos que el paciente nació de un parto normal y que presentó un desarrollo psicomotor normal en la primera infancia. Su hermano recordaba que con 4 años era un niño "ansioso" y que recibió "algún tipo de medicación" durante unos días. El paciente solía tomar prestados los juguetes de sus hermanos pero no prestaba los suyos. Acudió a centros escolares de cierto prestigio obteniendo buenos resultados académicos, aunque con bastante esfuerzo. Al parecer durante el tiempo de la escuela fue excluido por sus compañeros, que le tachaban de "peculiar". Completó estudios universitarios de Derecho y, a pesar de que se licenció, tardó 11 años en concluir esta licenciatura de cinco. Nunca trabajó como abogado. Se quedó en el domicilio familiar ayudando en distintas tareas. Cuando enfermó su abuelo fue su cuidador, y lo mismo hizo posteriormente con sus padres, hasta que fallecieron.

Su hermano le describió como "organizado, escrupuloso, prevenido y perfeccionista". Relató que solía tomar largas duchas y que era muy peculiar cuando guardaba cosas, teniendo que hacerlo de una manera específica. También había observado tics que incluían la nariz y cuello, y que el paciente tenía "desde siempre" un tic de carraspeo. No se le había conocido pareja sentimental.

Exploramos entonces al paciente, cuyo aspecto era ligeramente desaliñado, aunque se mostraba eutímico y con un afecto adecuado. Presentaba un tic en el brazo derecho, que movía como para recolocar su reloj. Su discurso era fluido y espontáneo, normal en el curso, pero extremadamente sobre-inclusivo y con acento gallego (de Galicia, región del norte de España). No se objetivaron síntomas en la esfera psicótica. Al explorar el contenido del pensamiento, explicaba que vivía "una vida simple y llana". Relató haber cuidado a su abuela y a sus padres, uno tras otro, y con ello justificaba no haber ejercido la abogacía. Refirió que no creía tener ningún problema previo a su ACV.

El hecho de que hablara con acento gallego era ciertamente inesperado ya que nunca había visitado dicha región ni tenía familiares o amigos naturales de allí.

Fue revalorado, junto a su hermano, 4 días más tarde. Admitió poseer unos 20.000 libros, 2.000 CDs y más de 2.000 DVDs en su piso. Relataba que solía comprarlos muy baratos, y que si veía uno que le gustara especialmente solía comprar 3 o 4 copias para poder regalar a familiares y amigos por Navidad o por cumpleaños. Se refería a su conducta acumuladora como una "estrategia financiera" para poder hacer regalos a un precio razonable. Lo mismo refería para los demás artículos encontrados en su piso. En ningún momento percibió su situación como un problema, dando justificaciones distintas, pero detalladas, para explicar su comportamiento. Negaba alteraciones senso-perceptivas. En la exploración cognitiva, puntuó 30/30 
en el MMSE y 92/100 en el ACEve (1) [versión española del Addenbrooke's Cognitive Examination; (2)], con una peor puntuación en memoria anterógrada (22/28) y fluencia verbal (6/7). Pudo explicar $3 / 3$ refranes y fue capaz de completar, con algún esfuerzo, el test de Luria.

Se realizó un diagnóstico de trastorno obsesivo compulsivo con tics crónicos y acumulación, así como síndrome del acento extranjero.

Se instauró un tratamiento psicofarmacológico a base de sertralina, con incremento progresivo hasta una dosis diaria de $150 \mathrm{mg}$, alcanzando una buena evolución. El discurso ya no resultaba sobre-inclusivo, el tic verbal había desaparecido, si bien mantenía el acento gallego. Siguió sin tener conciencia de su enfermedad, insistiendo en que su conducta era una "buena estrategia", aunque se acordó con el no comprar más artículos.

Se concertó una consulta de seguimiento tras 4 meses (6 meses después del ACV). Había permanecido estable psicopatológicamente. No se observaron tics, y el acento gallego - aunque seguía presente - apenas era perceptible. En la exploración cognitiva, puntúo 30/30 en el MMSE y 95/100 en el ACEve, habiendo perdido puntos en memoria anterógrada (27/28) y en fluencia verbal (6/7 para categorías y 4/7 para letras). Si bien seguía sin aceptar que padecía un TOC o una conducta acumuladora, aceptaba el tratamiento y el seguimiento. Vivía con un familiar y aunque aún no había ordenado su vivienda había acordado con su hermano comenzar a hacerlo tras alcanzar una recuperación física más completa.

Tras un año del ACV se objetivaron pocos cambios. El acento extranjero había desaparecido los tics seguían ausentes. Continuaba viviendo con un familiar y centraba sus esfuerzos en la "recuperación física", por lo que no había tenido tiempo para ordenar su piso, aunque acordó centrarse en ello tras recuperarse. La adherencia al tratamiento farmacológico era buena y acudía de forma regular a la consulta de su médico general, ya que no quiso vincularse al servicio de psiquiatría.

\section{Trastorno obsesivo compulsivo:}

El Trastorno obsesivo compulsivo (TOC) tiene una prevalencia a lo largo de la vida de 2 - $3 \%$ (3). Se caracteriza por la presencia de obsesiones y compulsiones. Las obsesiones se pueden entender como pensamientos, imágenes o impulsos intrusivos e indeseados que el sujeto reconoce como propios, a pesar de poseer un contenido perturbador e inquietante. Son frecuentes las ideas de contaminación, comprobación y de infligir daño a uno mismo y a terceros. El sujeto, al menos inicialmente, sí opone resistencia a estas experiencias intrusivas, aunque puede ceder ante ellas con el tiempo. Por otra parte, las compulsiones se definen como conduc- 
ORIGINALES Y REVISIONES

tas o actos mentales repetitivos y ritualistas, que se llevan a cabo en un intento de reducir la ansiedad generada por las obsesiones. Estos síntomas son angustiantes y crónicamente incapacitantes $(4,5)$. Un estudio longitudinal con seguimiento a 40 años señaló que aunque la mayoría de los pacientes muestran cierta mejoría con el tiempo, suelen presentar síntomas de manera crónica (6).

Los diversas experiencias subjetivas que preceden a las conductas de repetición (tics o compulsiones), son conocidas como "fenómenos sensoriales" y descritas como "sentimientos o sensaciones desagradables, focales o generalizadas, que preceden a la conducta de repetición y que habitualmente se ven aliviadas por ésta" (4). Trabajos posteriores han redefinido este término añadiendo la necesidad de realizar conductas repetitivas hasta que sienten que todo está "como debe ser" o "perfecto" (del inglés "just right"), conociéndose entonces como "percepciones de perfección". Otros autores han propuestos términos como "sensaciones corporales y mentales" (7); "impulsos premonitorios", "no perfección" o "incompleto" para describir estos fenómenos. Con el objetivo de alcanzar un consenso para la definición de estas experiencias, el equipo de Miguel aboga por mantener el término "fenómenos sensoriales" (8).

El TOC es un trastorno clínicamente heterogéneo. Los intentos de clasificar el trastorno en base a sus características clínicas, a menudo en subgrupos mutuamente excluyentes, han seguido dos enfoques distintos: categórico o dimensional. Los análisis factoriales de los distintos síntomas han respaldado las existencia de 4 factores dimensionales sintomáticos o expresiones fenotípicas del TOC: agresivo/comprobación, que incluye obsesiones sexuales, somáticas y religiosas (factor I); simetría/orden (factor II); contaminación/limpieza (factor III) y acumulación (factor IV). Estas dimensiones pueden solaparse en su presentación clínica, coexistiendo en cualquier paciente, y muestran estabilidad clínica a lo largo del tiempo (9-11).

Aunque la proporción hombre-mujer es de 1:1, se han encontrado diferencias entre géneros tanto en la sintomatología como en el curso de este trastorno. En los hombres se registran más antecedentes de sufrimiento perinatal, una edad de comienzo más temprana, más probabilidad de no haber estado casados, y una mayor frecuencia de obsesiones sexuales, de precisión y de simetría, así como de rituales extraños (factores I y II). Las mujeres, en cambio, mostraron una edad superior de comienzo del TOC, tenían más probabilidades de estar casadas al diagnóstico, presentaban obsesiones de contaminación/limpieza (factor III) y ataques de pánico, pero menor asociación con el trastorno bipolar $(12,13)$.

Investigaciones posteriores se han centrado en el estudio de la agregación familiar del TOC. Los resultados de los análisis de segregación coinciden en la implicación de "efectos genéticos dominantes" para los factores dimensionales I y II y de "efectos genéticos recesivos" para los factores dimensiónales III y IV (7). 
Las distintas dimensiones factoriales sintomáticas del TOC parecen estar asociadas a distintas vías neuroanatómicas dentro del circuito orbitofronto-estriado-talámico. Así, la "comprobación" (factor dimensional I) está asociada a la activación de putamen/globo pálido, tálamo y áreas corticales dorsales; la "aversión" (factor dimensional II) con regiones occipito-temporales izquierdas; el "limpìeza" (factor dimensional III) con regiones prefrontales ventromediales bilaterales y con el núcleo caudado izquierdo; y la "acumulación" (factor dimensional IV) está asociada al giro precentral izquierdo y la corteza orbitofrontal derecha (14).

\section{Trastorno por tic y TOC:}

Los tics son movimientos, gestos o sonidos repentinos y repetitivos, que típicamente imitan fragmentos de comportamientos normales. Paroxísticos y de duración breve, pueden darse también en racimos. Varían desde movimientos sencillos y abruptos hasta comportamientos con un objetivo. De la misma manera, los tics verbales o fónicos pueden oscilar entre un simple carraspeo a la coprolalia en los casos más severos (15).

El TOC con tics tiene características clínicas propias cuando se compara con el TOC sin tics. Suelen aparecer a una edad más temprana $(15,16)$, predomina en pacientes varones (aunque algunos autores no han constatado estas diferencias entre géneros) (17), está asociada con antecedentes familiares de trastorno por tics, tiene una base neuroquímica específica y responde a los ISRS con potenciación por neurolépticos. Los individuos que presentan un trastorno por tics crónico puntúan más alto en los factores dimensionales de agresividad/comprobación (I) y de simetría/orden (II) precedidos por cogniciones simultáneas y fenómenos sensoriales $(15,18-20)$. También se ha descrito un patrón anormal de excitabilidad cortical (21). Aunque la asociación TOC y tics es característica de otro cuadro, el Síndrome de Tourette (ST), este patrón también ha sido observado en pacientes con TOC y tics crónicos motores o verbales que no tienen (ST) (22).

Este fenotipo diferenciado del TOC, con una carga familiar mayor, parece estar relacionado con alteraciones del cromosoma 9p, que condicionan un genotipo diferente y frecuencias alélicas del alelo A del receptor serotoninérgico 5-HT2A $(7,23)$. 
ORIGINALES Y REVISIONES

\section{Comorbilidad psiquiátrica:}

Existe una prevalencia bien reconocida de trastornos psiquiátricos comórbidos al TOC. La literatura científica muestra que el 15\% de los pacientes inicialmente diagnosticados de TOC fueron posteriormente diagnosticados de esquizofrenia o trastorno bipolar. Éstos tienden a tener una edad de inicio más temprana y un peor pronóstico (4).

Hay una comorbilidad importante de fobias simples y fobia social, así como de Trastorno de pánico. Los pacientes con TOC experimentan niveles elevados de ansiedad, incluyendo ataques de pánico, que suelen ser secundarios a los pensamientos obsesivos. Un rasgo característico de estos ataques de pánico es que no son precipitados por la infusión de lactato (24).

El trastorno depresivo mayor es el cuadro psiquiátrico más frecuentemente asociado al TOC, con una prevalencia entre 17-70\%, según los distintos estudios. También se han comunicado casos de trastorno bipolar. Se destaca el hecho de que los síntomas del TOC típicamente mejoran durante los episodios de manía y empeoran durante los episodios depresivos $(4,25,26)$.

El trastorno de personalidad obsesivo-compulsivo (TPOC) puede confundirse con el TOC. Una diferencia notable reside en que los síntomas del TOC son egodistónicos, mientras que los de TPOC son egosintónicos y no existe la sensación de resistencia a la compulsión. Sólo entre 17\% y 25\% de los pacientes con TOC presentan un TPOC comórbido. Otros trastornos de personalidad que se han observado en sujetos con TOC incluyen los trastornos de personalidad evitativo, histriónico y dependiente (4).

\section{Modelos neuroanatómicos:}

A pesar de la implicación tan ampliamente aceptada de los ganglios basales en el TOC (27), los estudios de neuroimagen estructural han demostrado pocas alteraciones consistentes.

En la literatura científica se han descrito alteraciones tanto en sustancia gris como en sustancia blanca; éstas últimas han sido aún más ampliamente descritas (28). Recientemente, un estudio de imagen por tensores de difusión (DTI) ha detectado cambios en sustancia blanca en las regiones frontal medial derecha y parietal inferior derecha, tanto en pacientes con TOC como en familiares de primer grado (29). Por otra parte, las alteraciones en sustancia gris han sido descritas en los ganglios basales, córtex cingular anterior (CCA) y corteza prefrontal dorsolateral (CPFDL) (30). 
Comprensiblemente, el interés entorno a la neuroimagen funcional ha crecido. En estudios de PET recientes, aunque de forma inconsistente (31), se han detectado incrementos de la tasa metabólica cerebral de manera bilateral, especialmente en la cabezas de los núcleos caudados y en los giros orbitales de pacientes con TOC (32).

Estudios posteriores han mostrado anormalidades en el circuito orbito-fronto-estriatal $(5,33)$. Por ello, hasta la fecha, el modelo neuroanatómico se centra en alteraciones de las conexiones cortico-estriatales, concretamente en los circuitos orbito-fronto- estriado-talámicos. Se ha propuesto que la corteza orbito-frontal (COF) envía proyecciones neuronales a la cabeza del caudado y al estriado ventral, de ahí al globo pálido interno, tálamo mediodorsal, regresando a la COF. El hipocampo, cingulado anterior y amígdala basolateral también forman parte de este circuito, ya que están bien conectadas con la COF (5).

Más recientemente, se ha propuesto la existencia de un circuito neuronal diferente con implicación el la etiología del TOC. Coincidiendo con hallazgos previos (33), recientes meta-análisis de estudios de análisis a nivel de "voxel" han detectado alteraciones en el circuito orbito-fronto-estriatal, pero también han objetivado alteraciones por activación en corteza prefrontal lateral, cingulado anterior, estriado dorsal, cortezas occipital y parietal mediales y cerebelo, lo que apoya la implicación de la CPFDL en el TOC, que también se apoya en la disfunción cognitiva que se detecta en los pacientes con TOC. Los déficits en el "set shifting", planificación e inhibición de respuesta apoyan la participación de las cortezas CPFDL, prefrontalventrolateral (CPFVL) y parietal en el TOC $(5,30,34)$.

Este grupo de estudios propone la implicación de dos circuitos cerebrales en la etiología de TOC. Por un lado, el circuito orbito-fronto-estriatal (y las estructuras límbicas asociadas), que sería el "circuito afectivo"; y por otro el circuito prefronto-estriatal dorsolateral, que sería "el circuito espacial/atencional" (5). De esta manera, se propone ahora que el "circuito afectivo" estaría relacionado con los factores multi-dimensionales agresivo/comprobación (I) y contaminación/limpieza (III), mientras el circuito "espacial/atencional", involucrado en los déficits de inhibición de respuesta, estaría relacionado con los fenómenos "just right" y en el factor multi-dimensional simetría/orden (II) (35).

\section{Hallazgos cognitivos:}

En los pacientes con TOC se han objetivado déficits cognitivos muy diversos, especialmente en las funciones ejecutivas, memoria, y funciones verbales y psicomotrices, que, a su vez, se correlacionan con la severidad de los síntomas (36). 
ORIGINALES Y REVISIONES

La COF está implicada en la regulación emocional y conductual, y controla los cambios en el sistema de recompensa. Así se ha propuesto que tenga un papel específico en la inhibición de recompensa. La disfunción de la COF provoca déficits de aprendizaje relacionados con la recompensa, y por consiguiente alteraciones en el refuerzo de contingencias seguidas de cambios conductuales (5,37-40). Esto, a su vez, se traduce en una deficiencia en el comportamiento flexible y dirigido a un fin, dependiendo así los individuos de los mecanismos de control habituales, los hábitos "pre-aprendidos", que son desencadenados por estímulos específicos, a pesar de las consecuencias que puedan generar (41).

La implicación de la CPFDL, la CPFVL, la corteza cingulada e hipocampo contribuyen a disfunciones ejecutivas: en organización, a déficits en procesos de supervisión y a los déficits en la memoria de trabajo. Todo ello podría condicionar el mantenimiento de determinados comportamientos (como las compulsiones). Así, la disfunción ejecutiva se convierte en una característica clave del TOC $(37,40,42)$.

Algunas de estas características neuroanatómicas y cognitivas objetivadas en individuos con TOC están asociadas específicamente con una activación reducida de la COF lateral, CPF y cortezas parietales izquierdas durante el aprendizaje inverso, la rigidez cognitiva y los déficits de inhibición motora. Esto también se ha objetivado en familiares no afectados. De esta manera, el grupo de Cambridge propone estos hallazgos como prueba de la existencia de un fenotipo específico para el TOC $(29,38,43)$.

Consecuencias en la calidad de vida (del inglés Quality of life - QoL):

Existe consenso sobre la existencia de una QoL afectada en el TOC, informando incluso algunos autores sobre niveles inferiores a los objetivados en esquizofrenia.

Un estudio evaluó la QoL en sujetos con Trastorno de acumulación (TA) utilizando la versión breve de la Entrevista Lehman para Calidad de Vida (Lehman Quality of Life Interview-Short). Esta herramienta mide la QoL en diversos parámetros tales como: satisfacción vital general; situación vital; actividades diarias y funcionamiento ocupacional; familia; relaciones sociales; situación económica; trabajo y colegio; asuntos legales y de seguridad; y salud. Concluyó que los sujetos con TA mostraban una mayor afectación que aquellos con TOC en diversos ámbitos, pero especialmente en seguridad y situación de vivienda. Los sujetos con TA se sentían menos seguros en sus vecindarios, menos protegidos y más vulnerables al crimen (44). 


\section{Tratamiento:}

Muchos pacientes se benefician de un tratamiento son inhibidores selectivos de la recaptación de serotonina (ISRSs), pero el 10-40\% responden de manera incompleta. La edad de comienzo temprana, los síntomas compulsivos, los rituales de limpieza y el curso crónico con duración prolongada de la enfermedad son factores de mal pronóstico que indican una respuesta pobre a los ISRSs. La existencia de un trastorno de personalidad comórbido no influye en el pronóstico. Los casos en los que coexisten tics con el TOC se beneficiarían de la adición de un neuroléptico a los ISRSs (45).

Tanto los agentes serotoninérgicos como dopaminérgicos, no son eficaces en una proporción considerable de casos. Así, los nuevos enfoques terapéuticos se centran en el papel del glutamato, y su modulación, como posibilidad terapéutica en el tratamiento del TOC (46).

El glutamato es el neurotransmisor principal dentro del circuito cortico-estriado-talámico, siendo especialmente abundante en el estriado. De este modo, la literatura científica ha demostrado que la transmisión glutamatérgica está alterada en el TOC. Basándose en estudios de neuroimagen, algunos investigadores sugieren una disregulación de este circuito. Mientras por una parte existe una reducción tónica del glutamato en la ACC, por otra existe una hiperactividad glutamatérgica en el estriado y en la corteza OFC. Este hallazgo apunta a la posible acción terapéutica en el TOC de los agentes moduladores de glutamato (47).

El perfil conductual y cognitivo específico de los sujetos con TOC puede ser abordado mediante terapia conductual, la cual tendría también un papel claro en el manejo de este trastorno (48).

\section{Conclusiones:}

En resumen, el TOC es un trastorno heterogéneo que, aunque puede cursar con signos clínicos solapados, muestra estabilidad clínica en el tiempo. En un intento de alcanzar una clasificación del trastorno, se ha favorecido el enfoque multi-dimensional. Se han descrito alteraciones neuronales y cognitivas subyacentes cuya naturaleza precisa de futuras investigaciones para poder ser plenamente entendida. El TOC relacionado con tics parece ser una variante distinta de este trastorno. 
ORIGINALES Y REVISIONES

Acumulación: comportamiento o trastorno.

La acumulación ha sido descrita como "la adquisición o excesiva colección de objetos de poco valor y utilidad y la incapacidad para desecharlos ". Es característico que estos sujetos presenten déficit en el procesamiento de información, dificultades para establecer vínculos afectivos, conductas de evitación y creencias erróneas en relación con la naturaleza de los bienes que poseen (49).

Inicialmente, se consideraba que la acumulación era una conducta clínicamente significativa en menos del 5\% de los pacientes diagnosticados de TOC. Sin embargo, estudios más recientes sugieren que la conducta acumuladora se presenta comórbida al TOC en un 16\% al 35\% de los pacientes (50).

\section{Manifestaciones clínicas:}

La acumulación se caracteriza por la necesidad de almacenar objetos que en general no resultan de utilidad para la población general, y la incapacidad para desecharlos o deshacerse de ellos. Los niveles de angustia que genera la conducta en el paciente suelen ser inversamente proporcionales al grado de conciencia de enfermedad que presenten, siendo característico un escaso nivel de introspección o insight (51). Deberíamos cuestionarnos, entonces, si las personas con escasa capacidad de insight son "ciegas al desorden y la suciedad" en comparación con los que tienen una adecuada conciencia de enfermedad. Son necesarios futuros estudios que ayuden a responder a esta pregunta (52).

Las ideas obsesivas y la ansiedad autonómica solamente aparecen cuando la conducta de acumulación es prevenida $(4,51)$. La conducta de acumulación aparece en más del 30\% de pacientes TOC, por lo que los acumuladores constituyen un importante subgrupo de este trastorno (53). Se asocia a escasa respuesta al tratamiento (51), especialmente cuando la puntuación en el factor dimensional de acumulación (IV) es elevada (45). Sin embargo, cuando el tratamiento se adapta a las características clínicas del paciente y a los déficit asociados la efectividad del mismo mejora (54).

$\mathrm{Al}$ analizar la fenomenología de la acumulación compulsiva, se observa que difiere del TOC en que los pensamientos relacionados con la acumulación son percibidos por el sujeto, como normales, egosintónicos, no invasivos, no angustiosos ni desagradables. No son repetitivos como las ideas obsesivas, aunque tienden a empeorar con el tiempo (50). La excesiva adquisición de objetos a través de la compra compulsiva es común en los acumuladores con sentimientos positivos después de la compra, similares a los observados en trastorno por compra compulsiva (55). 
ORIGINALES Y REVISIONES

\section{Comorbilidad psiquiátrica:}

La conducta de acumulación se observa con mayor frecuencia en varones, sobre todo solteros, con una edad temprana de inicio, siendo rasgos característicos de estos pacientes el perfeccionismo y la indecisión. Las ideas obsesivas de contenido agresivo, sexual y religioso, los rituales de comprobación (factor dimensional I) y las ideas obsesivas de simetría y orden, junto a los rituales numéricos (factor dimensional II) son más frecuentes en este grupo de pacientes, mostrando mayor severidad de los síntomas TOC en la escala Y-BOCS. Sin embargo, menos del $20 \%$ de individuos que presentan trastorno de acumulación han sido previamente diagnosticados de TOC. La comorbilidad con otros trastornos psiquiátricos parece ser diferente en pacientes acumuladores y no acumuladores. Concretamente, el trastorno de ansiedad generalizada, los tics, la fobia social, la distimia, y el trastorno bipolar tipo II son más frecuentes en el grupo de acumuladores. El trastorno depresivo mayor es el trastorno psiquiátrico que mayor comorbilidad presenta con la conducta por acumulación, objetivándose junto a la fobia social y el trastorno de ansiedad generalizada en, aproximadamente, un $25 \%$ de los casos. Especialmente en las mujeres, se asocia a fobia social, trastorno por estrés postraumático, conductas higiénicas patológicas (impulsivas), rasgos dependientes de personalidad y baja meticulosidad. El trastorno obsesivo de personalidad y los trastornos de la personalidad cluster B, excepto el trastorno de personalidad antisocial, son también más frecuentes en este grupo de pacientes. Asimismo, se ha observado con mayor frecuencia la tendencia a la compra compulsiva, a la adquisición excesiva de artículos gratuitos y a la cleptomanía en los trastornos acumulativos que en los pacientes con TOC. Por otro lado, la conducta de acumulación se ha asociado a puntuaciones elevadas en cuestionarios auto-informados de cribado para el trastorno por déficit de atención e hiperactividad (TDAH). El riesgo de padecer TDAH es 10 veces mayor en los pacientes TOC con conductas de acumulación que en los TOC no acumuladores. El TDAH tipo con predominio de déficit de atención se ha evidenciado en casi el $30 \%$ de pacientes que presentan conductas de acumulación. Se agrupa en familias, siendo los familiares de primer grado más propensos a presentar conductas de acumulación $(53,55-58)$.

La conducta de acumulación también puede observarse en el contexto de un trastorno neuropsiquiátrico agudo, como el daño cerebral adquirido. En estos casos, los pacientes tienden a acumular comida y se muestran más desorganizados, con un patrón de adquisición más indiscriminado. Sin embargo, una parte de estos casos muestran una conducta verdaderamente directiva impulsada por las emociones que les generan los objetos. En estos casos, la presencia de otros síntomas neuropsiquiátricos, tales como el cambio de personalidad o los déficits cognitivos, orientará al clínico hacia el diagnóstico de conducta de acumulación secundaria a patología neurológica (59). 
ORIGINALES Y REVISIONES

\section{Genética:}

La acumulación compulsiva es una condición familiar, con factores genéticos que representan al menos el 50\% de dicha conducta (60). Los datos genotípicos muestran una significativa concordancia de alelos entre los acumuladores y el ST, observado en los cromosomas 5q, 4q y 17q. Dado que los cromosomas 4 y 17 están implicados en el ST, se propone que el cromosoma 5 podría ser un locus para el trastorno de acumulación (7). Estudios realizados en familias con sujetos diagnosticados de TOC, muestran que el cromosoma 14 está relacionado con la conducta de acumulación compulsiva. Es significativo que este hallazgo sólo se evidenció cuando la acumulación fue incluida en la definición fenotípica o en la estratificación de las familias de la muestra (61). Por lo tanto, los resultados de los estudios genéticos, aunque de manera inconsistente, apoyan la idea de que la acumulación es una entidad nosológica diferente del TOC (50).

\section{Hallazgo cognitivos:}

Cognitivamente la acumulación se asocia a un déficit en el procesamiento de la información, con dificultades con los vínculos afectivos y creencias distorsionadas acerca de las posesiones, y con las conductas de evitación y acumulación compulsiva específicas de este trastorno. Los acumuladores muestran creencias exageradas sobre sus posesiones, con un sentido de responsabilidad respecto a las mismas, y una excesiva valoración de la estética y los valores intrínsecos de dichos objetos. A nivel conductual, presentan conductas de evitación ante la posibilidad de desechar u organizar los objetos adquiridos, desinhibición (colección excesiva), una motivación limitada para el cambio, y no consideran su conducta como irracional (55).

En un estudio reciente, la evaluación neuropsicológica de los sujetos con trastorno de acumulación mostró que presentaban dificultades en la atención sostenida, con estrategias de planificación menos eficaces, pero sin objetivarse deterioro de la memoria verbal, de la no verbal, de las funciones ejecutivas, ni aumento de la impulsividad. Los autores de este estudio señalan que la falta de alteraciones en las funciones ejecutivas, puede deberse a un sesgo de selección de la muestra de individuos altamente motivados. Del mismo modo, concluyeron que los problemas observados en el proceso de toma de decisiones, eran secundarios a reacciones emocionales en relación con los objetos acumulados y no por deterioro neurocognitivo (62). Aún asumiendo que la disfunción ejecutiva propuesta sigue siendo un tema controvertido, parece que hay suficiente evidencia entorno a las dificultades que presentan los acumuladores en procesos de categorización (55), lo que tendría consecuencias a la hora de crear estrategias de descarte de los objetos acumulados. 


\section{Modelos neuroanatómicos:}

Utilizando técnicas de neuroimagen funcional, los investigadores han identificado un circuito fronto-límbico diferente al implicado en TOC, en el que participan de la corteza prefrontal ventromedial (CPFVM), la corteza orbitofrontal, la corteza cingulada y el sistema límbico. Por lo tanto, se sugiere que la disfunción de estas áreas interfiere en el control de las vías habituales para la adquisición y colección de objetos, así como en la percepción al deshacerse de objetos y posesiones personales, incluso sin valor, como un castigo $(59,63)$.

\section{Trastorno por acumulación: un trastorno por si mismo}

La evidencia encontrada hasta ahora, señala la conducta de acumulación como una manifestación clínica del TOC o como una entidad que difiere del Trastorno Obsesivo Compulsivo tanto en su modo de presentación y fenomenología, como en el perfil neuropsicológico y neuroanatómico.

En el DSM-III-R, la conducta de acumulación ya estaba catalogada como uno de los criterios de diagnóstico para el trastorno de personalidad obsesivo-compulsivo, sin embargo, no se incluye como criterio diagnóstico del trastorno anancástico de personalidad en la CIE-10. Según el DSM-IV-TR, cuando la acumulación es extrema, podría considerarse la presencia de un trastorno obsesivo compulsivo (TOC).

La literatura muestra que los pacientes con TOC que presentan conductas de acumulación, difieren en muchos aspectos de los pacientes con TOC no acumuladores. Expertos en la materia consideran que los acumuladores y los pacientes TOC con tics constituyen un grupo muy distinto al de otros pacientes TOC (35). De hecho, algunos investigadores consideran que en algunos pacientes la acumulación compulsiva constituye por sí misma un síndrome independiente al TOC, mientras que en otros pacientes la acumulación compulsiva es un síntoma del TOC de diferentes características psicopatológicas (64), algo que plantea interrogantes sobre los actuales criterios diagnósticos de dicho trastorno (57). Por este motivo algunos autores cuestionan incluso que la acumulación pueda considerarse un síntoma del TOC (65).

En este contexto, algunos estudios sugieren que la acumulación debe incluirse como una categoría independiente en el DSM-5, denominada como "Trastorno de acumulación", reservando el término "acumulación compulsiva" para los casos en los que la acumulación pueda considerarse un síntoma del trastorno obsesivo compulsivo $(50,59)$.

En la tabla 1 se exponen los criterios diagnósticos provisionales que el grupo de trabajo del DSM-5 ha descrito para el trastorno de acumulación. Destacan 
la importancia de diferenciar las conductas desorganizadas de acumulación, de la acumulación intencionada y el almacenamiento de objetos característico de la verdadera acumulación. La relación entre la acumulación y la suciedad también ha sido objeto de estudio, atribuyéndose la acumulación acompañada de suciedad a manifestaciones psiquiátricas de un trastorno del Eje I. La suciedad, en ocasiones extrema, y la acumulación desorganizada de elementos se considera consecuencia de un deterioro cognitivo secundario a anomalías estructurales o neurobiológicas, principalmente en la región fronto-temporal. Dado que únicamente una pequeña proporción de pacientes que presenta un trastorno de acumulación vive en condiciones de extrema suciedad y, ante la ausencia de evidencia a favor de que el trastorno de acumulación en presencia y ausencia de suciedad constituyan dos entidades diferenciadas, algunos autores cuestionan la inclusión de la suciedad como criterio diagnóstico en el DSM-5. Sin embargo, se recomienda registrar tanto la presencia o ausencia de suciedad, como el grado de la misma, ya que podría resultar relevante a la hora de establecer un plan de tratamiento y valorar el pronóstico del paciente, así como para plantear futuras líneas de investigación (52).

Tabla 1:

Propuesta de criterios diagnósticos para el trastorno de acumulación en el DSM-5. (F 02 Trastorno de Acumulación, American Psychiatric Association).

\footnotetext{
A. Dificultad persistente para desechar objetos o separarse de ellos, independientemente de su valor real.

B. Esta dificultad se debe a la necesidad percibida de guardar dichos elementos y el malestar asociado a deshacerse de ellos.

C. Los síntomas producen la acumulación de objetos que ocupan y abarrotan zonas de estar y sustancialmente comprometen su uso previsto. Que las zonas habitadas estén despejadas, suele ser únicamente debido a la intervención de terceros (por ejemplo, los miembros de la familia, limpiadores, autoridades).

D. La acumulación provoca malestar clínico significativo o deterioro tanto social, ocupacional como de otras áreas de funcionamiento (incluido el mantenimiento de un entorno seguro para el paciente y otros).

E. La acumulación no es atribuible a otra condición médica (por ejemplo, daño cerebral, enfermedad cerebrovascular, síndrome de Prader-Willi).

F. La acumulación no se explica mejor como síntoma de otro trastorno incluido en el DSM-5 (por ejemplo, la acumulación en relación con las obsesiones en el TOC, la anergia en el trastorno depresivo mayor, los delirios en la esquizofrenia u otros trastornos psicóticos, los déficits cognitivos en la demencia y los intereses restringidos en los trastornos del espectro autista).
}

\section{Especificar si:}

Con adquisición excesiva: Si los síntomas se acompañan de recolección excesiva o la compra o robo de artículos que no son necesarios o para los que no hay espacio disponible.

\section{Indicar si las creencias y conductas de acumulación se caracterizan actualmente por:}

Adecuada insight: el individuo reconoce que las creencias y conductas relacionadas con la acumulación (correspondiente a las dificultades para descartar elementos, el desorden, o la adquisición excesiva) son problemáticas.

Escasa insight: el individuo está en la mayor parte de las veces convencido de que las creencias y conductas relacionadas con la acumulación (correspondiente a las dificultades de descartar elementos, el desorden o la adquisición excesiva) no son problemáticas a pesar de la evidencia de lo contrario.

Ausencia de insight (es decir, las creencias delirantes acerca de la acumulación): el individuo está totalmente convencido de que las creencias y conductas relacionadas con la acumulación (correspondiente a las dificultades de descartar elementos, el desorden o la adquisición excesiva) no son problemáticas pesar de la evidencia de lo contrario. 
En un estudio publicado recientemente, se estima una prevalencia puntual del $5,8 \%$ para el trastorno de acumulación basándose en los criterios diagnósticos propuestos para el DSM-5. Los autores no objetivan diferencias significativas en relación al género e identifican el perfeccionismo, la indecisión y el aplazamiento como características asociadas a la conducta de acumulación (66). Los resultados de otro estudio muestran una prevalencia entre el $2 \%$ y el $5 \%$ y, señalan que el trastorno de acumulación podría ser el doble de frecuente que el TOC (67). Es inevitable entonces cuestionarse por qué el trastorno de acumulación no se diagnostica con mayor frecuencia y qué implicaciones podría tener para la salud pública en el futuro.

\section{Tratamiento:}

Suele resultar complicado implicar a los individuos con Trastorno de Acumulación en cualquier tipo de tratamiento, debido a su falta de insight y a la resistencia que muestran a eliminar el desorden y limpiar su casa. Un grupo de investigadores sugiere involucrar al acumulador y a su familia a través de la entrevista motivacional para minimizar el daño que produce esta patología (68).

Aunque el tratamiento farmacológico del Trastorno de Acumulación sigue siendo motivo de controversia, algunos investigadores apoyan el uso de la paroxetina o venlafaxina (forma de liberación prolongada) como una alternativa terapéutica eficaz, mostrando la venlafaxina una menor tasa de abandono del tratamiento (69).

\section{Conclusiones:}

Las evidencias reunidas hasta ahora sugieren que la acumulación compulsiva es un síntoma del TOC, mientras que el Trastorno de Acumulación ha sido ahora aceptado como una entidad nosológica con sus propias manifestaciones clínicas distintivas, su fenomenología, su perfil neuropsicológico y su sustrato neurológico. El Trastorno de Acumulación aparece en el DSM-5 como un trastorno en sí mismo, informando los estudios preliminares de una mayor prevalencia que el TOC. 
ORIGINALES Y REVISIONES

\section{Síndrome del acento extranjero}

El síndrome del acento extranjero (SAE) es un raro trastorno motor del habla que fue descrito por primera vez por Pierre Marie en 1917 (70), dos años más tarde por Pick (71), pero quizás es más conocida la descripción de Monrad-Kröhn a finales de los años 1940 (72,73).

Esencialmente consiste en que, debido a las distorsiones en la articulación del lenguaje, el discurso es percibido por los nativos parlantes como con un acento extranjero.

El SAE se considera una apraxia del habla. La terminología utilizada inicialmente incluía términos como "disartria cortical" o "afasia atáxica", debido a que existen dificultades en la motricidad necesarias para pronunciar los fonemas adecuadamente (74). Sin embargo, algunos investigadores creen que el SAE se puede distinguir de la disartria o la apraxia del habla debido a que éstas implican una articulación laboriosa. En su lugar, señalan que las alteraciones en la prosodia verbal, tal y como describió Monrad-Kröhn, causan la percepción de un acento extranjero dentro de la producción de un discurso normal, no disártrico ni apráxico (75). Sin embargo, se acepta que tanto los déficits en la prosodia como en la producción del lenguaje son esenciales para la producción de FAS (76).

Con frecuencia, pero no siempre, el "acento extranjero" aparece después de un período de afasia, cuando el sujeto recupera el habla $(75,77)$.

A pesar de que normalmente aparece después de un ACV o daño cerebral adquirido, también ha sido descrita en los síntomas iniciales de la afasia primaria progresiva, en la esclerosis múltiple, como resultado de una metástasis cerebral, en el contexto de trastornos psiquiátricos tales como el trastorno afectivo bipolar, los trastornos de conversión o de etiología poco clara con estudios de neuroimagen no concluyentes (78-83).

Esta patología se asocia a lesiones cerebrales en el hemisferio dominante del lenguaje, en la corteza motora, en la corteza de asociación motora frontal y en el núcleo estriado o región paravermal del cerebelo, mientras que algunos autores señalan una disfunción de las vías de retroalimentación cortico-subcortical como factor contribuyente a la aparición de este síndrome $(74,84,85)$.

Encontramos un informe sobre un caso de remisión espontánea del SAE 3 años después del debut (76), aunque la remisión espontánea es rara y difícil de encontrar en la literatura en casos de "acento extranjero" que persiste durante años. Muy poco se sabe acerca de su tratamiento mediante terapia del habla y el lenguaje (73). 
ORIGINALES Y REVISIONES

\section{Discusión}

Nuestro paciente, de acuerdo a su presentación y a la información recopilada, comenzó a mostrar síntomas de TOC a una edad temprana. Se trata de un hombre joven que no se ha casado y que mostró síntomas que se pueden incluir en el factor multidimensional II (simetría y obsesiones en cuanto al orden y compulsiones de conteo) y IV (acumulación). También presentó diferentes tics tanto motores como verbales. Él no refiere "alteraciones sensoriales" y nosotros no pudimos observarlas durante las diferentes consultas quellevamos a cabo. Como características premórbidas, el paciente fue descrito como "organizado, escrupuloso, prevenido, y perfeccionista", mostrando rasgos compatibles con una personalidad anancástica.

Presentó una clara conducta de acumulación, recogiendo miles de libros y DVDs, viviendo en un piso abarrotado, pero al mismo tiempo en un ambiente limpio. Sabía cuántos artículos tenía, ya que los había organizado a través de una lista completa de objetos. El paciente no mostró ningún pensamiento obsesivo respecto a su conducta de acumulación, mostrándose ansioso solamente cuando el estado de su piso fue descubierto y, en un principio, cuando comenzaron los comentarios sobre la limpieza del piso y la redistribución de sus pertenencias. El paciente fue capaz de dar, a su modo de ver, una explicación válida y lógica de su conducta, obviando la evidencia de que tendría que tener miles de amigos y familiares para que su explicación tuviera sentido, de modo que mostraba una escasa capacidad de insight.

En los tests cognitivos realizados no mostró alteraciones cognitivas groseras, sino un leve deterioro en la fluencia verbal y en la memoria anterógrada. Su pensamiento abstracto estaba intacto $\mathrm{y}$, sin embargo, era evidente la rigidez cognitiva en relación con su conducta de acumulación, no estando dispuesto a entretener la posibilidad que su comportamiento pudiera ser difícil de entender para los demás.

Después de su ACV, no se constata un período de afasia, ni por el paciente ni por sus familiares. Su discurso era gramaticalmente correcto, no se objetivaba disartria pero mostró un claro acento gallego que fue percibido por el personal, familiares, otros pacientes y los familiares de éstos. Creemos que también es de interés el que su acento recién adquirido desapareciera 6 meses después de la lesión cerebral inicial. El paciente no mostró ninguna otra alteración psicopatológica, que la descrita en este documento, y su comportamiento no nos hizo sospechar un trastorno psicogénico que pudiera ser responsable de su aparición. Tampoco nos pareció que su aparición fuera compatible con el diagnóstico de síndrome de Tourette.

Nosotros creemos que el daño en el núcleo estriado fue la causa de la aparición del SAE, y tan sólo podemos postular que la regeneración y plasticidad neuronal después de la lesión inicial son los responsables de su desaparición.

Curiosamente, que nosotros sepamos, es el único caso de TOC en su familia. En ausencia de carga genética para el TOC y su conducta de acumulación, acepta- 
ORIGINALES Y REVISIONES

mos que una explicación plausible de su etiología sería una interrupción del circuito fronto-estriado. Teniendo en cuenta que el paciente sufrió una hemorragia en el núcleo estriado izquierdo, sólo podemos suponer que presentó una disfunción preACV de este circuito, quizá en el núcleo estriado, que fue responsable del TOC, los tics y la conducta de acumulación.

También es reseñable que el paciente respondió bien al tratamiento con un inhibidor específico de la recaptación de serotonina (ISRS), sin que se llegara a asociar un neuroléptico. El tic desapareció y el paciente estuvo dispuesto a dejar de adquirir nuevos artículos y redistribuir los materiales almacenados con la ayuda de sus familiares.

\section{CONCLUSIONES}

Creemos que este caso es una prueba más de que diferentes entidades nosológicas comparten el mismo circuito neuronal.

Aceptamos que si bien desde una edad temprana el paciente presentó signos clínicos compatibles con el diagnóstico de TOC, asociados a tics motores y verbales, fue su conducta de acumulación lo que adquirió un significado clínico relevante. Creemos que esto refleja una superposición de síntomas clínicos multidimensionales en el TOC que pueden cambiar en el tiempo, con la acumulación, en este caso, como una manifestación clínica más del TOC.

A nuestro entender, este es el primer caso descrito de la variante regional de SAE en una persona de habla española y también de un paciente que presenta comorbilidad con TOC, tics y la conducta de acumulación. A su vez, no somos conocedores de ningún otro caso de SAE con remisión espontánea 6 meses después del ACV inicial.

Figura 2

Imagen de una resonancia magnética nuclear (RMN): imágenes de un T1 sagital y un T2 axial mostrando un hematoma crónico con un grado de degeneración circundante.
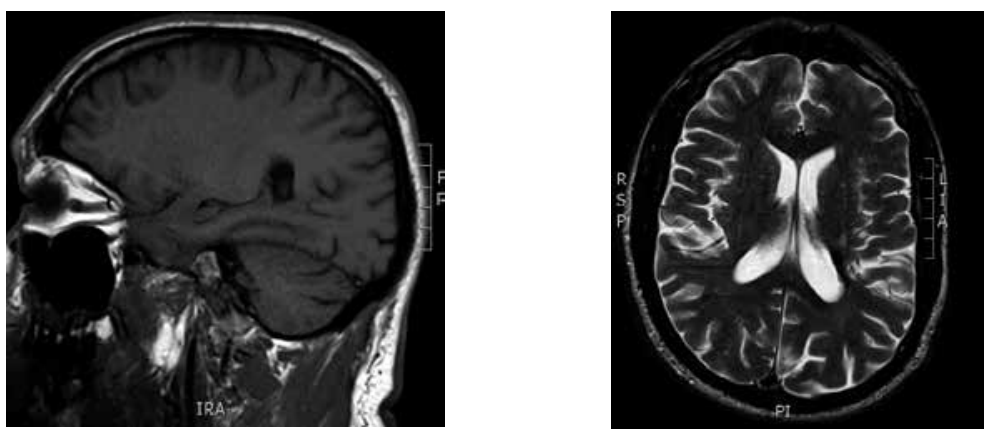
ORIGINALES Y REVISIONES

\section{BIBLIOGRAFÍA:}

(1) García Caballero A, García Lado I, González Hermida J, Recimil MJ, Area R, Manes F, Lamas $\mathrm{S}$ et al. Validation of the Spanish version of the Addenbrooke's Cognitive Examination in a rural community in Spain. International Journal of Geriatric Psychiatry 2006; 21: 239-245.

(2) Mathuranath PS, Nestor PJ, Berrios GE, Rakowicz W, Hodges JR. A brief cognitive test battery to differentiate Alzheimer's disease and frontotemporal dementia. Neurology 2000; 55: 16131620.

(3) Karno M, Golding JM, Sorenson SB, Burnam MA. The epidemiology of obsessivecompulsive disorder in five US communities. Archives of General Psychiatry 1988; 45: 1094-1099.

(4) Miguel EC, Rauch SL, Jenike MA. Obsessive-Compulsive Disorder in Neuropsychiatry of the Basal Ganglia. The Psychiatric clinics of North America 1997; 20(4): 863-883.

(5) Menzies L, Chamberlain SR, Laird AR, Thelen SM, Sahakian BJ, Bullmore ET. Integrating evidence from neuroimaging and neuropsychological studies of obsessive-compulsive disorder: The orbitofronto-striatal model revisited. Neuroscience and Biobehavioral Reviews 2008; 32: 525-549.

(6) Skoog G, Skoog I. A 40 year follow up of patients with obsessive-compulsive disorder. Archives of General Psychiatry 1999; 56: 121-127.

(7) Miguel EC, Leckman JF, Rosario-Campos MC, Hounie AG, Mercadante MT et al. Obsessive-compulsive disorder phenotypes: implications for genetic studies. Molecular Psychiatry 2005; 10: 258-275.

(8) Prado HS, Rosario-Campos MC, Shavitt RG, Miguel EC. Sensory phenomena, "just-right” and "not just-right" experiences in OCD patients: looking for a consensus. CNS Spectrums 2007; 12(2): 95-96.

(9) Leckman JF, Grice DE, Boardman J, Zhang H, Vitale A, Bondi C et al. Symptoms of Obsessive-compulsive disorder. American Journal of Psychiatry 1997; 154: 911-917.

(10) Mataix-Cols D, Rosario-Campos MC, Leckman JF. A multidimensional model of obsessivecompulsive disorder. American Journal of Psychiatry 2005; 162: 228-238.

(11) Mataix-Cols D. Deconstructing obsessive-compulsive disorder: a multidimensional perspective. Current Opinion in Psychiatry 2006; 19: 84-89.

(12) Lensi P, Cassano GB, Correddu G, Ravagli S, Kunovac JL, Akiskal HS. Obsessivecompulsive disorder. Familial-developmental history, symptomatology, comorbidity and course with special reference to gender-related differences. British Journal of Psychiatry 1996; 169: 101-107.

(13) Labad J, Menchon JM, Alonso P, Segalas C, Jimenez S, Jaurrieta N et al. Gender differences in obsessive-compulsive symptom dimensions. Depression and Anxiety 2008; 25: 832-838.

(14) Mataix-Cols D, Wooderson S, Lawrence N, Brammer MJ, Speckens A, Phillips ML. Distinct neural correlates of washing, checking and hoarding symptom dimensions in obsessive-compulsive disorder. Archives of General Psychiatry 2004; 61: 564-576.

(15) Leckman JF, Peterson BS, Pauls DL, Cohen DJ. Tic disorders in Neuropsychiatry of the Basal Ganglia. The Psychiatric clinics of North America 1997; 20(4): 840-861.

(16) Janowitz D, Grabe HJ, Ruhrmann S, Ettelt S, Buhtz F, Hochrein A et al. Early onset of obsessive-compulsive disorder and associated comorbidity. Depression and Anxiety 2009; 26: 1012-1017.

(17) Rosario-Campos MC, Leckman JF, Mercadante MT, Shavitt RG, Prado HS, Sada P et al. Adults with early-onset obsessive-compulsive disorder. American Journal of Psychiatry 2001; 158: 18991903. 
ORIGINALES Y REVISIONES

(18) MilletB,KochmanF,GallardaT,KrebsMO,DemonfauconF,BarrotIetal.Phenomenological and comorbid features associated in obsessive-compulsive disorder: influence of age of onset. Journal of Affective Disorders 2004; 79: 241-246.

(19) Zohar AH, Pauls DL, Ratzoni G, Apter A, Dycian A, Binder M et al. Obsessive-compulsive disorder with and without tics in an epidemiological sample of adolescents. American Journal of Psychiatry 1997; 154: 274-276.

(20) Storch EA, Stigge-Kaufman D, Marien WE, Sajid M, Jacob ML, Geffken GR et al. Obsessive-compulsive disorder in youth with and without a chronic tic disorder. Depression and Anxiety 2008; 25: 761-767.

(21) Greenberg BD, Ziemann U, Corá-Locatelli G, Harmon BS, Murphy DL, Keel JC et al. Altered cortical excitability in obsessive-compulsive disorder. Neurology 2000; 54(1): 142-147.

(22) Diniz BJ, Rosario-Campos MC, Hounie AG, Curi M, Shavitt RG, Lopes AC et al. Chronic tics and Tourette syndrome in patients with obsessive-compulsive disorder. Journal of Psychiatric Research 2006; 40: 487-493.

(23) MiguelEC,BaerL, Coffey BJ, Rauch SL, Savage CR, O’Sullivan RLet al.Phenomenological differences appearing with repetitive behaviours in obsessive-compulsive disorder and Gilles de la Tourette's syndrome. British Journal of Psychiatry 1997; 170: 140-145.

(24) Gorman JM, Liebowitz MR, Fyer AJ, Dillon D, Davies SO, Stein J et al. Lactate infusions in obsessive-compulsive disorder. American Journal of Psychiatry 1985; 142: 864-866.

(25) Gordon A, Rasmussen SA. Mood-related obsessive-compulsive symptoms in a patient with bipolar affective disorder. Journal of Clinical Psychiatry 1988; 49: 27-28.

(26) Nestadt G, Di CZ, Riddle MA, Grados MA, Greenberg BD, Fyer AJ et al. Obsessivecompulsive disorder: subclassification based on co-morbidity. Psychological Medicine 2009; 39 14911501 .

(27) Rapoport JL, Wise SP. Obsessive-compulsive disorder: evidence for basal ganglia dysfunction. Psychopharmacology Bulletin 1988; 24: 380-384.

(28) Jenike MA, Breiter HC, Baer L, Kennedy DN, Savage CR, Olivares MJ et al. Cerebral structural abnormalities in obsessive-compulsive disorder: a quantitative morphometric magnetic resonance imaging study. Archives of General Psychiatry 1996; 53(7): 625-632.

(29) Menzies L, Williams GB, Chamberlain SR, Ooi C, Fineberg N, Suckling J et al. White matter abnormalities in patients with obsessive-compulsive disorder and their first degree relatives. American Journal of Psychiatry 2008; 165: 1308-1315.

(30) Radua J, Mataix-Cols D. Voxel-wise meta-analysis of grey matter changes in obsessive compulsive disorder. British Journal of Psychiatry 2009; 195: 393-402.

(31) Aylward EH, Harris GJ, Hoehn-Saric R, Barta PE, Machlin SR, Pearlson GD. Normal caudate nucleus in obsessive-compulsive disorder assessed by quantitative neuroimaging. Archives of General Psychiatry 1996; 53(7): 577-584.

(32) Baxter Jr LR, Schwartz JM, Mazziota JC, Phelps ME, Pahl JJ, Guze BH et al. Cerebral glucose metabolic rates in non depressed patients with obsessive-compulsive disorder. American Journal of Psychiatry 1988; 145: 1560-1563.

(33) Breiter HC, Rauch SL, Kwong KK, Baker JR, Weisskoff RM, Kennedy DN et al. Functional magnetic resonance imaging of symptom provocation in obsessive-compulsive disorder. Archives of General Psychiatry 1996; 53(7): 595-606. 
(34) Harrison BJ, Soriano-Mas C, Pujol J, Ortiz H, López-Solá M, Hernández-Ribas R et al. Altered corticostriatal functional connectivity in obsessive-compulsive disorder. Archives of General Psychiatry 2009; 66(11): 1189-1200.

(35) Leckman JF, Bloch MH. A developmental and evolutionary perspective on obsessivecompulsive disorder: whence and whither compulsive hoarding? American Journal of Psychiatry 2008; 165: 1229-1233.

(36) Abramovitch A, Dar R, Schweiger A, Hermesh H. Neuropsychological impairments and their association with obsessive-compulsive symptom severity in obsessive-compulsive disorder. Archives of Clinical Neuropsychology 2011; 26: 364-376.

(37) Rauch SL, Savage CR. Neuroimaging and neuropsychology of the striatum in Neuropsychiatry of the Basal Ganglia. The Psychiatric Clinics of North America 1997; 20(4): 741-768.

(38) Chamberlain SR, Menzies L, Hampshire A, Suckling J, Fineberg NA, del Campo N et al. Orbitofrontal dysfunction in patients with obsessive-compulsive disorder and their unaffected relatives. Science 2008; 321: 421-422.

(39) Gharemani DG, Monterosso J, Jentsch D, Bilder RM, Poldrack RA. Neural components underlying behavioural flexibility in human reversal learning. Cerebral Cortex 2010; 20: 1843-1852.

(40) Rygula R, Walker SC, Clarke HF, Robbins TW, Roberts AC. Differential contributions of the primate ventrolaterral prefrontal and orbitofrontal cortex to serial reversal learning. The Journal of Neuroscience 2010; 30(43): 14552-14559.

(41) Gillan CM, Papmeyer M, Morein-Zamir S, Sahakian BJ, Fineberg NA, Robbins TW et al. Disruption in the balance between goal-directed behavior and habit learning in obsessive-compulsive disorder. American Journal of Psychiatry 2011; 168: 718-726.

(42) Van den Heuvel OA, Veltman DJ, Groenewegen HJ, Cath DC, van Balkom AJLM, van Hartskamp J et al. Frontal-striatal dysfunction during planning in obsessive-compulsive disorder. Archives of General Psychiatry 2005; 62: 301-310.

(43) Chamberlain SR, Fineberg NA, Menzies LA, Blackwell AD, Bullmore ET, Robbins TW et al. Impaired cognitive flexibility and motor inhibition in unaffected first degree relatives of patients with obsessive-compulsive disorder. American Journal of Psychiatry 2007; 164: 335-338.

(44) Saxena S, Ayers CR, Maidment KM, Vapnik T, Wetherell JL, Bystritsky A. Quality of life and functional impairment in compulsive hoarding. Journal of Psychiatric Research 2011; 45: 475-480.

(45) Mataix-Cols D, Rauch SL, Manzo PA, Jenike MA, Baer L. Use of factor-analyzed symptom dimensions to predict outcome with serotonin reuptake inhibitors and placebo in the treatment of obsessivecompulsive disorder. American Journal of Psychiatry 1999; 156: 1409-1416.

(46) Pittenberg C, Bloch MH, Williams K. Glutamate abnormalities in obsessive compulsive disorder: Neurobiology, pathophysiology, and treatment. Pharmacology \& Therapeutics 2011; 132: 314-332.

(47) Wu K, Hanna GL, Rosenberg DR, Arnold PD. The role of glutamate signalling in the pathogenesis and treatment of obsessive-compulsive disorder. Pharmacology Biochemistry and Behavior 2012; 100: 726-735.

(48) Beck AT. The current state of cognitive therapy. A 40 year perspective. Archieves of General Psychiatry 2005; 62: 953-959.

(49) Frost RO, Hartl TL. A cognitive-behavioural model of compulsive hoarding. Behavior Research \& Therapy 1996; 34(4): 341-350.

(50) Mataix-Cols D, Frost RO, Pertusa A, Clark LA, Saxena S, Leckman JF et al. Hoarding disorder: A new diagnosis for DSM-5? Depression and Anxiety 2010; 27: 556-572. 
ORIGINALES Y REVISIONES

(51) Black DW, Monahan P, Gable J, Blum N, Clancy G, Baker P. Hoarding and treatment response in 38 non-depressed subjects with obsessive compulsive disorder. Journal of Clinical Psychiatry 1998; 59: 420-425.

(52) Snowdon J, Pertusa A, Mataix-Cols D. On hoarding and squalor: a few considerations for DSM-5. Depression and Anxiety 2012; 29: 417-424.

(53) Samuels J, Joseph Bienvenu III O, Riddle MA, Cullen BAM, Grados MA, Liang K-Y et al. Hoarding in obsessive-compulsive disorder: results from a case-control study. Behaviour Research and Therapy 2002; 40: 517-528.

(54) Saxena S, Maidment KM, Vapnik T, Golden G, Rishwain T, Rosen RM et al. Obsessivecompulsive hoarding: symptom severity and response to multimodal treatment. Journal of Clinical Psychiatry 2002; 63(1): 21-27.

(55) Tolin DF. Understanding and treating hoarding: A biopsychosocial perspective. Journal of Clinical Psychology: In session 2011; 67: 517-526.

(56) Samuels JF, Joseph Bienvenu O, Pinto A, Murphy DL, Piacentini J, Rauch SL et al. Sexspecific clinical correlates of hoarding in obsessive-compulsive disorder. Behaviour Research and Therapy 2008; 46:1040-1046.

(57) Saxena S. Is compulsive hoarding a genetically and neurobiologically discrete syndrome? Implications for diagnostic classification. American Journal of Psychiatry 2007; 164(3): 380-384.

(58) Frost RO, Steketee G, Tolin DF. Comorbidity in hoarding disorder. Depression and Anxiety 2011; 28: 876-884.

(59) Mataix-Cols D, Pertusa A, Snowdon J. Neuropsychological and neural correlates of hoarding: a practice friendly review. Journal of Clinical Psychology: In session. 2011; 67: 467-476.

(60) Iervolino AC, Perroud N, Fullana MA, Guipponi M, Cherkas L, Collier DA et al. Prevalence and heritability of compulsive hoarding: a twin study. American Journal of Psychiatry 2009; 166: 11561161 .

(61) Samuels J, Shugart YY, Grados MA, Willour VL, Joseph Bienvenu O, Greenberg BD et al. Significant linkage to compulsive hoarding on chromosome 14 in families with obsessive-compulsive disorder: results from the OCD collaborative genetics study. American Journal of Psychiatry 2007; 164 : 493-499.

(62) Tolin DF, Villavicencio A, Umbach A, Kurtz MM. Neuropsychological functioning in hoarding disorder. Psychiatry Research 2011; 189: 413-418.

(63) Tolin DF, Kiehl KA, Worhunsky P, Book GA, Maltby N. An exploratory study of the neural mechanisms of decision making in compulsive hoarding. Psychological Medicine 2009; 39: 325-336.

(64) Pertusa A, Fullana MA, Singh S, Alonso P, Menchón JM, Mataix-Cols D. Compulsive hoarding: OCD symptom, distinct clinical syndrome, or both? American Journal of Psychiatry 2008; 165 : 1289-1298.

(65) Abramowitz JS, Wheaton MG, Storch EA. The status of hoarding as a symptom of obsessive compulsive disorder. Behaviour Research and Therapy 2008; 46: 1026-1033.

(66) Timpano KR, Exner C, Glaesmer H, Rief W, Keshaviah A, Brähler E et al. The epidemiology of the proposed DSM-5 hoarding disorder: Exploration of the acquisition specifier, associated features, and distress. Journal of Clinical Psychiatry 2011; 72(6): 780-786.

(67) Tolin DF. Challenges and advances in treating hoarding. Journal of Clinical Psychology: In session. 2011; 67: 451-455. 
(68) Tompkins MA. Working with families of people who hoard: a harm reduction approach. Journal of Clinical Psychology: In session 2011; 67: 497-506.

(69) Saxena S. Pharmacotherapy of compulsive hoarding. Journal of Clinical Psychology: In session. 2011; 67: 477-484.

(70) Marie P, Foix C. Les aphasies de guerre. Rev. Neurol (Paris) 1917; 24: 53-87.

(71) Pick A. Ueber aenderungen des sprachcharakters als begleitersheinung aphasischer strörungen. Zeitschrift fuer de gesamte Neurun Psychologie 1919; 54: 230-241.

(72) Monrad-Kröhn GH. Dysprosodia or altered "melody of language”. Brain 1947; 70: 405415.

(73) González-Álvarez J, Parcet-Ibars MA, Ávila C, Geffner-Sclarsky D. Una rara alteración del habla de origen neurológico: el síndrome del acento extranjero. Revista de Neurología 2003; 36(3): 227234.

(74) Mariën P, Verhoeven J, Engelborghs S, Rooker S, Pickut BA, De Deyn PP. A role for the cerebellum in motor speech planning: Evidence from foreign accent syndrome. Clinical Neurology and Neurosurgery 2006; 108: 518-522.

(75) Takayama Y, Sugishita M, Kido T, Ogawa M, Akiguchi I. A case of foreign accent syndrome without aphasia caused by a lesion of the left pre-central gyrus. Neurology 1993; 43: 1361-1363.

(76) Berthier ML. Foreign accent syndrome. Neurology 1994; 44: 990-991.

(77) Naidoo R, Warriner EM, Oczkowski WJ, Sévigny A, Humphreys KR. A case of foreign accent syndrome resulting in regional dialect. The Canadian Journal of Neurological Sciences 2008; 35 : 360-365.

(78) Abel TJ, Hebb AO, Silbergeld DL. Cortical stimulation mapping in a patient with foreign accent syndrome: case report. Clinical Neurology and Neurosurgery 2009; 111: 97-101.

(79) Gurd JM, Coleman JS, Costello A, Marshall JC. Organic or functional? A new case of foreign accent syndrome. Cortex 2001; 37: 715-718.

(80) Luzzi S, Viticchi G, Piccirilli M, Fabi K, Pesallaccia M, Bartolini M et al. Foreign accent síndrome as the initial sign of primary progresive aphasia. Journal of Neurology Neurosurgery and Psychiatry 2008; 79: 79-81.

(81) Poulin S, Macoir J, Paquet N, Fossard M, Gagnon L. Psychogenic or neurogenic origin of agrammatism and foreign accent syndrome in a bipolar patient: a case report. Annals of General Psychiatry 2007; 6:1.

(82) Villaverde-González R, Fernández-Villalba E, Moreno-Escribano A, Alías-Linares E, García-Santos JM. Síndrome del acento extranjero como primera manifestación de esclerosis múltiple. Revista de Neurología 2003; 36(11): 1035-1039.

(83) Verhoeven J, Mariën P, Engelborghs S, D’Haenen H, De Deyn P. A foriegn speech accent in a case of conversion disorder. Behavioural Neurology 2005; 16: 225-232.

(84) Karanasios P, Loukopoulou P,Zampakis P, Tiligadas T, Makidrou A, Doukas V et al. Foreign accent syndrome caused by a left temporal-parietal ischaemic stroke. Acta Neuropsychiatrica 2011; 23: 249-251.

(85) Cohen DA, Kurowski K, Steven MS, Blumstein SE, Pascual-Leone A. Paradoxical facilitation: the resolution of foreign accent syndrome after cerebellar stroke. Neurology 2009; 73(7): 566567. 